Revista de Investigación Universitaria, 2015, Vol. 4 (2): 71-78

ISSN: 2312-4253 (Versión impresa) ISSN: 2078-4015 (Versión digital)

\title{
Propiedades Psicométricas del Inventario de Depresión de Beck en estudiantes de 5 to año de secundaria de Huancayo, 2013
}

\section{Psychometric Properties of the Beck Depression Inventory in High School Students of Huancayo, 2013}

\author{
Rodríguez Amaro, Eder Apolos; Farfán Rodríguez, Daniel \\ EP. Psicología, Facultad de Ciencias Empresariales, Universidad Peruana Unión \\ Recibido 10 de setiembre del 2015-Aceptado 26 de noviembre del 2015
}

\begin{abstract}
Resumen
El objetivo de la investigación fue determinar las propiedades psicométricas del inventario de Depresión de Beck (BDI-IIA), en un grupo de 300 adolescentes varones y mujeres de 10 instituciones educativas estatales de la provincia de Huancayo. La edad de los participantes fue entre los 16 y 18 años. La validez de constructo fue evaluada por medio del análisis factorial exploratorio y confirmatorio. Los resultados obtenidos señalan que el modelo propuesto inicialmente, que consiste en dos factores cognitivo-afectivo y somático-motivacional, también es confirmado en el presente estudio. Asimismo, se observa un total de alfa de Cronbach de .891. Además, buenos índices de ajuste, saturaciones elevadas e índices de consistencia interna adecuados para ambas dimensiones. Por lo cual se concluye que el inventario posee adecuados niveles de fiabilidad y validez en la muestra estudiada.
\end{abstract}

Palabras clave: Depresión, cognitivo- afectivo, somático- motivacional, adolescentes

\begin{abstract}
The aim of the research was to determine the psychometric properties of the Beck Depression Inventory (BDIIIA) in a group of 300 male and female adolescents from 10 State Educational Institutions in the province of Huancayo. The age of participants was between 16 and 18 years. The construct validity was evaluated through exploratory and confirmatory factor analysis. The results obtained indicate that the initially proposed model, which consists of two cognitive-affective and Somatic-motivational factors, is also confirmed in the present study. Also, a total of Cronbach's alpha of .891 is observed. In addition, good indexes of fit, high saturations and indices of internal consistency suitable for both dimensions. Therefore, it is concluded that the inventory has adequate levels of reliability and validity in the sample studied.
\end{abstract}

Keywords: Depression, cognitive-affective, motivational- somatic, adolescent

Correspondencia al autor:

email: eder24_05@hotmail.com 


\section{Introducción}

La adolescencia es una etapa de cambios significativos, esto conlleva a pensar que se trata de una población de alta vulnerabilidad. En general, todas las personas somos vulnerables a distintos problemas sociales y personales que pueden desembocar en enfermedades físicas y mentales. Es así que, en ocasiones, en un intento frustrado de la búsqueda de soluciones se originan los trastornos que aquejan el estado de ánimo, como es el caso de la depresión.

Autores como Beck (1983) consideran a la depresión como un trastorno del estado de ánimo, donde el individuo es atrapado por pensamientos distorsionados, que ponen en riesgo su salud psíquica y que en el caso de los adolescentes, como diría Stanley Hall (véase en Papalia y Olds, 1996), sufren un período de tormenta y estrés que los hace más vulnerables en su adaptación al medio.

La evaluación de la depresión, en adolescentes, puede realizarse a través de múltiples instrumentos. Sin embrago, la mayoría de estos carece de una traducción y estandarización que se adapte a poblaciones específicas.

En el Perú el BDI-II (Beck Depression Inventory) es un instrumento usado casi exclusivamente para la población clínica, y los estudios que usan este instrumento tienen como objetivo describir la sintomatología depresiva o la correlación de variables.

El BDI-IIA ofrece una sólida base teórica, que incluye los nueve síntomas depresivos del DSM-IV (American Psychiatric Association [APA], 1995), no obstante, la traducción y estandarización de este inventario no se ha difundido y estudiado tanto como su predecesora, respecto a sus propiedades psicométricas.

Respecto a la estandarización del BDI-IIA, para la población adolescente de la localidad de Huancayo, no se hallaron estudios previos, pese a una alta tasa epidemiológica de casos de depresión publicada por la Dirección Regional de Salud - Junín, donde se observa un incremento del $20 \%$ en el quinquenio 20052009, comparado con el quinquenio anterior (Samaniego, Gonzales, Calle \& Calle, 2009)

Entonces, la necesidad de contar con instrumentos de evaluación estandarizados, en nuestro medio, para la detección de trastornos depresivos que permita al evaluador una adecuada identificación del trastorno, se hace evidente.

\section{Método}

\section{Diseño}

El presente estudio es de tipo instrumental, según la clasificación realizada por Montero y León (2005) en vista que el estudio tiene como propósito indagar las propiedades psicométricas donde se manifiestan una o más variables. Además, se trata de una investigación transversal, ya que se busca recopilar datos en un solo momento (Hernández, Fernández, Baptista, 2006).

\section{Participantes}

Se realizó un muestreo no probabilístico de tipo intencional, en el cual se obtuvo una muestra piloto de 150 alumnos (70 varones y 80 mujeres). Los sujetos encuestados pertenecían a colegios estatales de 5to año de secundaria de la ciudad de Huancayo y sus edades oscilaban entre 16 y 18 años de edad. Posteriormente se obtuvo una muestra grande representada por 300 alumnos (120 varones y 180 mujeres) de 16 y 18 años, del 5to año de educación secundaria de diez instituciones estatales de la ciudad de Huancayo.

\section{Instrumentos}

La versión original del inventario de depresión de Beck (BDI) se introdujo en 1961 por Beck, Ward, Meldenson, Mack y Erbaug como una prueba autoadministrada, después de un tiempo, en 1971, Beck y sus colaboradores emplean la versión modificada del BDI en el centro de terapia cognitiva de la Universidad de Pennsylvania. Eliminaron algunas palabras y frases alternativas para los mismos síntomas evitando la doble negación, más tarde, en 1987, se publicó el manual técnico para el inventario de depresión de Beck adaptado (BDI-IA). Sin embargo, la última publicación del inventario de Beck (BDI- II) se realizó en 1996, con la intensión de valorar la depresión y derivarla a observaciones clínicas en pacientes psiquiátricos. El BDI-II posee una alta consistencia interna, alta validez de contenido, especificad en la identificación de pacientes deprimidos y sanos.

En relación a sus propiedades psicométricas, el BDIII ha mostrado una buena consistencia interna (alfa de Crombach 0.88), asimismo, la validez de constructo que se obtuvo, a través de la técnica de análisis fac- 
torial, son resultados con resultados altos útiles para acreditar el BDI-II como un instrumento válido.

Beck et al. (1996) observaron una alta consistencia interna del BDI-II, tanto en muestras clínicas como no clínicas, con un coeficiente alfa de alrededor de 0,92. En la adaptación española, realizada por el equipo de Sanz, el Inventario para la Depresión de Beck-II obtuvo niveles elevados de consistencia interna tanto en muestras de universitarios, de población general y de consultantes con trastornos psicopatológicos Sanz y García (2013); Sanz, Navarro y Vásquez (2003); Sanz, Perdigón y Vásquez (2003).

El Inventario de Depresión, de Beck-Segunda Versión BDI-II, es un instrumento de autoaplicación de 21 ítems. Ha sido diseñado para evaluar la gravedad de la sintomatología depresiva en adultos y adolescentes con una edad mínima de 13 años. En cada uno de los ítems, la persona tiene que elegir entre un conjunto de cuatro alternativas ordenadas de menor a mayor gravedad, la frase que mejor describe su estado durante las últimas dos semanas, incluyendo el día en que completa el instrumento. En cuanto a la corrección, cada ítem se valora de 0 a 3 puntos en función de la alternativa escogida y, tras sumar directamente la puntuación de cada ítem, se puede obtener una puntuación total que varía de 0 a 63 .
Para la presente investigación se utilizó BDI-IIA (Inventario de Depresión de Beck Adaptado). Recientemente Carranza (2011) realizó un estudio con estudiantes universitarios de la ciudad de Lima y cuyas propiedades psicométricas describimos a continuación. En cuanto a la validez de contenido del instrumento, fue estudiado inicialmente a través de la validez por criterio de jueces. Para cuantificar los hallazgos se aplicó el coeficiente V de Aiken (Escurra, 1998). Asimismo, el BDI-IIA presentó un índice de alfa de Cronbach de 0.878, lo cual indica que el instrumento presenta una consistencia interna óptima.

\section{Resultados}

\section{Análisis de índice de homogeneidad}

La Tabla 1 muestra el análisis de correlaciones entre las dimensiones obtenidas que evidencia una correlación significativa $(\mathrm{p}<.001)$ y positiva entre dichos ítems, con valores superiores a $r>0.5$, para ambas dimensiones (cognitivo-afectivo y somático- motivacional), sin embargo, los ítems 7. Y 14. (7. disconformidad con uno mismo y 14. desvalorización) fueron añadidos a la dimensión cognitivo-afectivo por estar relacionados con dicho factor, con un peso factorial para ítem 7 de .595 y el ítem 14. de .664.

Tabla 1

Índice de homogeneidad de medición de los ítems a través de las correlaciones de Pearson.

\begin{tabular}{lll}
\hline \multicolumn{1}{c}{ Factor Cognitivo-Afectivo } & \multicolumn{1}{c}{ Factor Somático- motivacional } \\
\hline Tristeza &, $588^{* *}$ Agitación &, $547^{* *}$ \\
Pesimismo &, $617^{* *}$ Pérdida de interés &, $611^{* *}$ \\
Fracaso &, $665^{* *}$ Indecisión &, $616^{* *}$ \\
Pérdida de placer &, $627^{* *}$ Pérdida de energía &, $675^{* *}$ \\
Sentimiento de culpa &, $620^{* *}$ Cambio en los hábitos de sueños &, $619^{* *}$ \\
Disconformidad con uno mismo &, $595^{* *}$ Irritabilidad &, $604^{* *}$ \\
Autocrítica &, $721^{* *}$ Cambios de apetito &, $645^{* *}$ \\
Pensamientos o deseos de suicida &, $693^{* *}$ Dificultad de concentración &, $651^{* *}$ \\
Llanto &, $570^{* *}$ Fatiga &, $701^{* *}$ \\
Desvalorización &, $664^{* *}$ & \\
\hline
\end{tabular}

\section{Análisis de fiabilidad}

El análisis de la consistencia interna (ver Tabla 2) de cada una de las dimensiones ofreció los siguientes resultados. En la dimensión cognitivo-afectivo se muestra un alfa de Cronbach de .837, el cual indica que el instrumento presenta un alto índice que fiabilidad según Huh, Delorme y Reid (2006) quienes proponen que el valor de fiabilidad en estudios confirmatorios debe estar entre 7 y .8 .

Para la dimensión somático-motivacional, se muestra un alfa de Cronbach de .808, respaldando al instrumento aplicado, por lo que los resultados evidencian niveles de fiabilidad satisfactorios, para el total de la escala y las correspondientes dimensiones. 
Tabla 2

Análisis de fiabilidad de los items y homogeneidad de los subescalas (cognitivo-afectivo y somático- motivacional)

\begin{tabular}{lc}
\hline Dimensiones & Alpha de Cronbach $(\alpha)$ \\
\hline Subescala cognitivo-afectivo & 0,837 \\
Subescala somático-motivacional & 0,808 \\
Depresión Total & 0,891 \\
\hline
\end{tabular}

\section{Análisis factorial confirmatorio}

Mediante el análisis factorial exploratorio se detectaron 2 ítems (ítems 6 y 21) que presentaron valores bajos, por esta razón se procedió a la eliminación de estos dos ítems, pues presentan puntajes inferiores a .5, ya que la teoría señala que para que el ítem sea aceptado, el valor general de las correlaciones tiene que ser $\geq .5$ (George \& Mallery, 2003).

Además, se evaluó el ajuste de modelo de dos factores latentes, el factor latente correspondiente a cognitivo-afectivo está indicado por 10 ítems (1, 2, 3, 4,5, $7,8,9,10,14)$ y el factor somático-motivacional está indicado por 9 ítems (11, 12, 13, 15, 16, 17, 18, 19, 20). Los resultados del análisis factorial confirmatorio demuestran, en la Tabla 3, que el índice de $\mathrm{X}^{2}$ presenta un ajuste global perfecto $\left(\mathrm{X}^{2}=210.898\right)$.
Infiriéndose que el modelo propuesto, en la figura 1 , es estadísticamente significativo pues el ajuste de bondades adecuado.

Asimismo, el modelo de los dos factores relacionados presentó un buen ajuste (ver tabla 3); donde el GFI (índice de bondad de ajuste) $=.931$, presenta un valor superior a .90 y el RMSEA (raíz del residuo cuadrático promedio de aproximación) $=0.036$, arroja un valor próximo a cero, indicando que ambos valores demuestran que el modelo presenta un alto grado, en el cual el modelo estimado predice la matriz de datos.

Por otro lado, el NFI (índice de ajuste normalizado) $=.875$, y el CFI (índice de bondad de ajuste comparativo) $=.961$ también declara que nuestro modelo tiene un buen ajuste y el AIC (criterio de información de Aiken $)=288.898$, no presenta resultados conflictivos, por lo que es menor al modelo de independencia y al saturado. Y por último, el índice de error RMR (raíz del residuo cuadrático promedio) $=0.027$ evidencia un valor reducido que apoya al buen ajuste del modelo, según Fleming (1985). Analizando los resultados y confirmando el modelo propuesto para estudiantes del nivel secundario para provincia, sería la que se muestra en la figura 1.

Tabla 3

Análisis factorial confirmatorio del cuestionario de depresión de Beck

\begin{tabular}{lccccccccc}
\hline \multicolumn{1}{c}{ Dimensiones } & $\mathbf{X}^{2}$ & Gl & AIC & NFI & CFI & GFI & AGFI & RMR & RMSEA \\
\hline \multicolumn{1}{c}{ Depresión } & 210.898 & 151 & 288.898 & .875 & .961 & .931 & .913 & 0.027 & 0.036 \\
\hline $\mathrm{P}<0.01$ & & & & & & & & &
\end{tabular}

En la figura 1 se muestra el modelo como resultado del análisis factorial confirmatorio que propone dos dimensiones: cognitivo-afectivo y somático-motivacional, propuesto también por Sanz, Perdigón, Vásquez (2003). Respecto a los indicadores, estos obtienen pesos moderados y altos, mientras que la correlación entre los dos factores es de .84.

\section{Distribución de variables final}

La Tabla 4 presenta la distribución de los ítems finales del cuestionario de depresión de Beck, donde los ítems 1, 2, 3, 4, 5, 7, 8, 9, 10, 14 forman parte de la dimensión cognitivo- afectivo y los ítems 11, 12, 13, $15,16,17,18,19,20$, forman parte de la dimensión somático-motivacional. 
Tabla 4

Distribución de variables e items final del cuestionario de Depresión de Beck.

\begin{tabular}{|c|c|c|}
\hline Variable & Dimensión & Ítems \\
\hline DEPRESIÓN & Cognitivo-afectivo & $\begin{array}{ll}\text { 1. } & \text { Tristeza } \\
\text { 2. } & \text { Pesimismo } \\
\text { 3. } & \text { Fracaso } \\
\text { 4. } & \text { Pérdida del placer } \\
\text { 5. Sentimientos de culpa } \\
\text { 7. Disconformidad con uno mismo } \\
\text { 8. Autocritica } \\
\text { 9. Pensamientos o deseos suicidas } \\
\text { 10. Llanto } \\
\text { 14. Desvalorización } \\
\text { 11. Agitación } \\
\text { 12. Pérdida de interés } \\
\text { 13. Indecisión } \\
\text { 15. Pérdida de energía } \\
\text { 16. Cambios en los hábitos de sueño } \\
\text { 17. Irritabilidad } \\
\text { 18. Cambios de apetito } \\
\text { 19. Dificultad de concentración } \\
\text { 20. Cansancio o fatiga }\end{array}$ \\
\hline
\end{tabular}

\section{Baremos Finales}

Para establecer los baremos para el nuevo modelo del instrumento, se consideró la misma proporción del instrumento inicial y, por medio de los resultados de los percentiles, los baremos quedaron establecidos de la siguiente manera (ver Tabla 5 y 6 ).

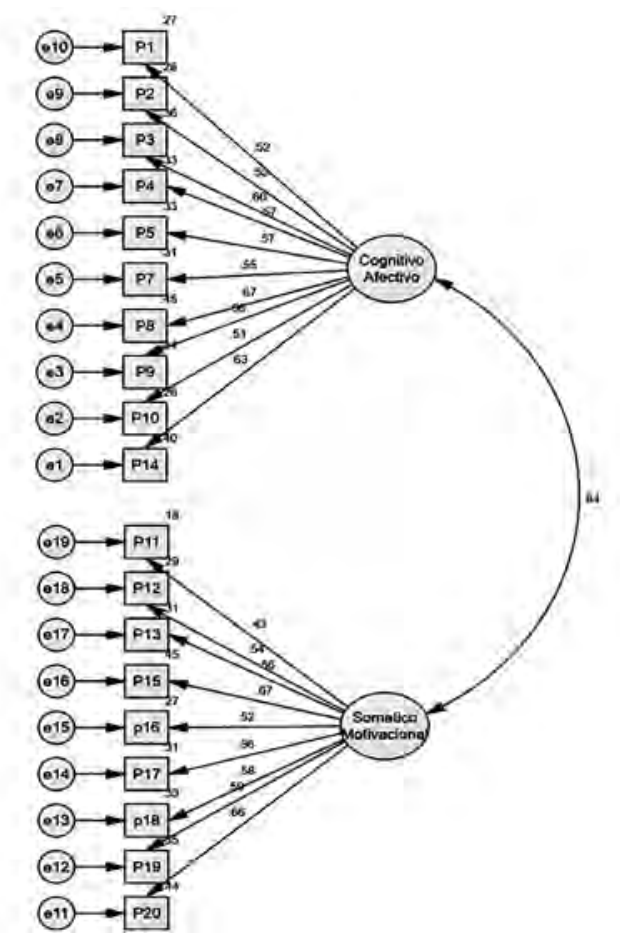

Figura 1. Modelo de análisis factorial confirmatorio. 


\section{Percentiles}

Para la categorización se utilizaron los siguientes niveles: minino, leve, moderado y severo, tras sumar directamente la puntuación de cada ítem se obtuvo una puntuación total que varía de 0 a57. Respecto a la suma de ítems por dimensiones, se obtuvo una puntuación de 0 a 30 para la dimensión cognitivo-afectivo y la suma de ítems de 0 a 27 para la dimensión somático-motivacional

Tabla 5

Percentiles de las dimensiones y la depresión

\begin{tabular}{cccc}
\hline Percentiles & Cognitivo-afectivo & Somático-motivacional & Depresión \\
\hline Válidos & 300 & 300 & 300 \\
Perdidos & 0 & 0 & 0 \\
25 & 3,0000 & 6,0000 & 10,0000 \\
50 & 7,0000 & 9,0000 & 15,0000 \\
75 & 11,0000 & 11,0000 & 21,0000 \\
\hline
\end{tabular}

Tabla 6

Puntajes directos categorizados para evaluar los niveles de depresión

\begin{tabular}{lll}
\hline Cognitivo-afectivo & Total & Somático-motivacional \\
\hline 0-3 Mínimo & 0-10 Mínimo & 0-6 Mínimo \\
4-7 Leve & 11-15 Leve & 7-9 Leve \\
8-11 Moderado & 16-21 Moderado & 10-11Moderado \\
12-30 Severo & 22-57 Severo & 12-27 Severo \\
\hline
\end{tabular}

\section{Discusión}

Uno de los objetivos que tenía la presente investigación era explorar la validez de la prueba a través del análisis factorial exploratorio (AFE) y confirmatorio (AFC). El número de factores obtenidos era similar a lo hallado en otras investigaciones (Sanz, Perdigón \& Vásquez 2003; Carranza, 2011) que lograron obtener una estructura de dos factores. Sin embargo, es necesario señalar que estos modelos (Sanz, Perdigón \& Vásquez 2003; Carranza, 2011) presentaban una distribución de los ítems diferente a la hallada en el modelo de la presente investigación. Estos resultados parecen indicar que la estructura factorial del BDIIIA no muestra un constructo sólido.

Otra posible explicación, de esta variabilidad observada en los modelos obtenidos en las diferentes adaptaciones, podría indicar poca consistencia en la estructura factorial de la prueba. Ya que, si bien en este estudio el tamaño de la muestra puede estar influyendo en la solución hallada, las investigaciones en las que no se han reproducido la estructura factorial planteada por la prueba han contado con muestras de mayor tamaño (Sanz, Perdigón \& Vásquez 2003; Carranza, 2011; Melipillán, Cova, Rincón \& Valdivia,
2008) lo cual parece indicar que los factores del BDIIIA, no muestran un constructo sólido.

También se podría inferir que la dificultad para replicar la estructura factorial, señalada por los autores que iniciaron las investigaciones con el cuestionario, es producto de las características de la muestra, por ejemplo: poca capacidad de introspección, deseabilidad social (necesidad de quedar bien con el investigador), escasa comprensión lectora, etc.

Con el objetivo de optimizar el funcionamiento de la prueba en la muestra algunos ítems fueron eliminados. A continuación, se formularán respuestas en relación a las dificultades que han podido presentar estos reactivos.

Si se tiene en cuenta el modelo original propuesto por Beck (2006) y estudiado por Carranza (2011), donde el factor cognitivo-afectivo agrupa los ítems 1, 2, 3, 4, 5, 8, 9, 10 y el factor somático-motivacional agrupa los ítems 6, 7, 11, 12, 13, 14, 15, 16, 17, 18, 19, 20, 21, notamos que son dos los ítems se procedieron a eliminar. Estos son dentro de la dimensión somático-motivacional, el ítem 6. (Sentimiento de castigo) con un coeficiente de .42 y el ítem 21. (Pérdida de 
interés por el sexo) con un coeficiente de .47 los mismos que no se ajustan de manera adecuada a ninguna variable (George \& Mallery, 2003).

Asimismo, el ítem 7 (disconformidad con uno mismo) y 14 (desvalorización), con un coeficiente de .454 y .563 respectivamente, y que en el diseño inicial se relacionaba con la dimensión somático-motivacional, fueron trasladados a la dimensión cognitivo-afectivo por estar relacionados con dicho factor, con un peso factorial de .595 para ítem 7. y .664 para el ítem 14.

Otro objetivo, de esta investigación, fue analizar la confiabilidad del BDI-IIA a partir de la consistencia interna de los reactivos para cada uno de los factores que componen la prueba. Los resultados obtenidos indican que el BDI-IIA cuenta con una confiabilidad aceptable.

De otro lado, los resultados del análisis factorial confirmatorio muestran, en la Tabla 10, que el índice de $\mathrm{X}^{2}$ indica un ajuste global, perfecto siendo $\mathrm{X}^{2}$ $=210.898$. Infiriéndose que el modelo propuesto final (ver figura 1) es estadísticamente significativo, pues el ajuste de bondad es adecuado.

Por otro lado, si se tiene en cuenta el valor del alfa de Cronbach hallado en la presente investigación .891, en contraste a otras investigaciones, se halla que por ejemplo que Melipillán, Cova, Rincón y Valdivia (2008) hallaron un alto grado de consistencia interna (alfa $=0,91$ ), similar al alfa de Cronbach de la presente investigación y una apropiada correlación test-retest $(\mathrm{t}=0,66)$.

Asimismo, Sanz, Perdigón, Vásquez (2003) quienes presenta por primera vez datos normativos de fiabilidad y validez factorial de la adaptación española del BDI-II, encontraron la fiabilidad de consistencia interna elevada (coeficiente alfa de 0,87).

Del mismo modo, Beltrán y Freyre (2012) quienes estudiaron el inventario de depresión de Beck y su validez en una población adolescente de México hallaron un alfa de Cronbach $=.92$.

Además, podemos contrastar los hallazgos de la presente investigación con los de Carranza (2011), dado que los índices de confiabilidad en la presente investigación son similares. Aunque, por otro lado, la confiabilidad hallada por Sanz et al. (2003) es menor a la que se encontró aquí.
Este último aspecto sobre la diferencia entre la confiabilidad hallada por Sanz et al. (2003) y los resultados de confiabilidad de la presente investigación pueden ser explicados por Prieto y Delgado (2010), quienes refieren que la variabilidad de los índices de fiabilidad, entre poblaciones, se debe a las características de cada población, por ejemplo: idiosincrasia, sistema de valores, etc. En consecuencia, se ha de evitar el error de considerar que la estimación de la fiabilidad procedente de un único estudio, refleja la verdadera y única fiabilidad de la prueba.

Finalmente, los índices de homogeneidad, hallados en la presente investigación, están por encima de .50 en todas las escalas, y son coherentes a los valores sugeridos por George y Mallery (2003), exceptuando el ítem 6 (sentimiento de castigo), con un coeficiente de .42 y el ítem 21 (pérdida de interés por el sexo), con un coeficiente de .47 .

\section{Referencias}

American Psychiatric Association (1995). DSM-IV-TR. Manual diagnostico y estadístico de los trastornos mentales. Cuarta Edición. Texto revisado. Barcelona: Masson.

Beck, A., Steer, R., y Brown, G. (1996). BDI-II, Beck Depression inventory: Manual (2nd ed.). Boston: Harcourt Brace.

Beck, A. (1983). Terapia cognitiva de la depresión. Bilbao, Desclee de Brouwer.

Beck, A., Steer, R., Brown, G., (2006). Inventario de depression de Beck. Argentina: Paidós

Beavers, S.; Lounsbury, W.; Richards, K.; Huck, W., Skolits, J. y Esquivel, L. (2013). Practical considerations for using exploratory factor analysis in educational research, Practical Assessment, Research and Evaluation. 18(6)

Beltrán, M. Freyre, M. (2012). El inventario de Depresión de Beck: Su validez en población adolescente. Terapia psicológica. 30, 5-13.

Carranza, R. (2011). Propiedades psicométricas de inventario de Beck para universitarios de Lima. Tesis para optar el título en psicología. Lima, Perú: Universidad Peruana Unión.

Escurra, L. (1998). Cuantificación de la validez de contenido por criterio de jueces. Revista de psicología, Pontificia Universidad Católica del Perú, 6 (2), 103- 105. 
Fleming, J. (1985). An Index of Fit for Factor Scales. Educational and Psychological Measurement, 45, 725-728

George, D. y Mallery, M. (2003). Usando SPSS para Windows paso a paso: Una guía y referencia simple. Boston, MA: Allyn y Bacon.

Hernández, R., Fernández, C. y Baptista, P. (2006). Metodología de la investigación México: Mc Graw-Hill.

Huh, J., Delorme, D. y Reid, L. (2006). Efectos percibidos en tercera y actitudes de consumo sobre la prevención y prohibición de la publicidad DTC. Diario de asuntos del consumidor. 40, 90-91.

Kehoe, J. (1995). Análisis de ítems básicos para las pruebas de selección múltiple. Evaluación práctica, investigación y evaluación. 4(10)

Montero, I. y León, O. (2005). Sistema de clasificación del método en los informes de investigación en psicología. International Journal of Clinical and Health Psychology, 5, 115-127.

Melipillán, R., Cova, F., Rincón, P. y Valdivia, M. (2008). Propiedades Psicométricas del Inventario de Depresión de Beck-II en Adolescentes Chilenos. Revista Terapia Psicológica de Chile, 26(1), 59- 69.
Papalia, D., Olds, S. (1996). Psicología del Desarrollo. De la infancia a la adolescencia. México: Mc Graw Hill.

Prieto, G. y Delgado, A. (2010). Fiabilidad y Validez. En papeles del psicólogo, 31(1), 67-74.

Samaniego, C., Gonzales, E., Calle, M., Calle, I. (2009). Relación entre el estado afectivo y el rendimiento académico en los estudiantes de la institución Educativa Mariscal Castilla. Tesis de enfermería Huancayo, Perú. Tesis de la facultad de enfermería de la Universidad Nacional del Centro del Perú.

Sanz, J., Perdigón, A. y Vázquez, C. (2003). Adaptación española del Inventario para la Depresión de Beck-II (BDI-II): 2. Propiedades psicométricas en población general. Clínica y Salud, 14, 249-280.

Sanz, J., Navarro, M. y Vázquez, C. (2003). Adaptación española del Inventario para la Depresión de Beck-II (BDI-II): 1. Propiedades psicométricas en estudiantes universitarios. Análisis y Modificación de Conducta, 29, 239-288.

Sanz, J. y García, P. (2013). Rendimiento diagnóstico y estructura factorial del inventario de Depresión de Beck. Anales de Psicología, 29, 66-75. 